\title{
Characterizing underlying cognitive components of ADHD presentations and co-morbid diagnoses - A diffusion decision model analysis
}

\author{
Nadja R. Ging-Jehli ${ }^{1}$, L. Eugene Arnold ${ }^{2}$, Michelle E. Roley-Roberts ${ }^{3}$, Roger deBeus ${ }^{4}$ \\ ${ }^{1}$ Department of Psychology, The Ohio State University \\ ${ }^{2}$ Department of Psychiatry and Behavioral Health, Nisonger Center UCEDD, The Ohio \\ State University \\ ${ }^{3}$ Department of Psychiatry, Creighton University \\ ${ }^{4}$ University of North Carolina at Asheville, Asheville, NC
}

Corresponding author: Nadja R. Ging-Jehli (ORCID: 0000-0002-1071-0693), Department of Psychology, 1835 Neil Avenue Mall, The Ohio State University, Columbus, OH 43210. nadja@gingjehli.com

Manuscript accepted for publication in the Journal of Attention Disorders. Re-use restricted to non-commercial and no derivative uses. This is a preprint and not the copy of record and may not exactly replicate the authoritative document that will be published in the Journal of Attention Disorders.

\section{Declarations}

Funding: This research was supported by Swiss National Science Foundation \#P1SKP1_184033; National Institute of Mental Health grant \#R01-MH100144, by Ohio State University College of Medicine Endowment, and by Clinical and Translational Science award 8UL18TR000090-05 from the National Center for Translational Sciences. Conflicts of interest: N.R. Ging-Jehli received research funding from SNSF and neurocognitive tests from testmakers. L.E. Arnold received research funding from Curemark, Forest, Lilly, Neuropharm, Novartis, Noven, Shire, Supernus, Roche, and YoungLiving (as well as NIH and Autism Speaks), has consulted with Gowlings, Neuropharm, Organon, Pfizer, Sigma Tau, Shire, Tris Pharma, and Waypoint, and been on advisory boards for Arbor, Ironshore, Novartis, Noven, Otsuka, Pfizer, Roche, Seaside Therapeutics, Sigma Tau, Shire. M.E. Roley-Roberts has no potential conflict of interest pertaining to this Journal of Abnormal Child Psychology submission. R. deBeus received research funding from NIMH; he is on the Board of Directors for the International Society for Neurofeedback and Research and has a clinic in NC where he performs neurofeedback and other clinical services. Ethics approval: The study was approved by Institutional Review Board. N.R. Ging-Jehli received research funding from SNSF and neurocognitive tests from testmakers. All participants provided written consent prior to participation. Acknowledgment: N.R. Ging-Jehli thanks SNSF for support of her graduate studies. She also thanks: Russ Childers for insightful discussions; Roger Sandford for the neurocognitive tests; Roger Ratcliff for providing access to modeling scripts; Catherine Panchyshyn, Rachel Bergman, Arielle Cottrell, Alex Lingel, Madeline Thomas, Shea Connor for invaluable help with data collection. 


\begin{abstract}
Objective. To Explore whether subtypes and comorbidities of attention-deficit hyperactivity disorder (ADHD) induce distinct biases in cognitive components involved in information processing. Method. Performance on the Integrated Visual and Auditory Continuous Performance Test (IVA-CPT) was compared between 150 children (aged 7-10 years) with ADHD, grouped by DSM-5 presentation (ADHD-C, ADHD-I) or co-morbid diagnoses (anxiety, oppositional defiant disorder [ODD], both, neither), and 60 children without ADHD. Diffusion decision modeling decomposed performance into cognitive components. Results. Children with ADHD had poorer information integration than controls. Children with ADHD-C were more sensitive to changes in presentation modality (auditory/visual) than those with ADHD-I and controls. Above and beyond these results, children with ADHD+anxiety+ODD had larger increases in response biases when targets became frequent than children with ADHD-only or one comorbidity. Conclusion. ADHD presentations and comorbidities have distinct cognitive characteristics quantifiable using DDM and IVA-CPT. We discuss implications for tailored cognitive-behavioral therapy.
\end{abstract}




\section{Introduction}

The Diagnostic and Statistical Manual of Mental Disorders (DSM-5; American Psychiatric Association [APA], 2013) distinguishes among three presentations of attention-deficit hyperactivity disorder (ADHD) based on whether the predominant symptom is inattention (ADHD-I); hyperactivity-impulsivity (ADHD-H); or a combination of both inattention and hyperactivity-impulsivity (ADHD-C). Moreover, children with ADHD frequently struggle not only with inattention and/or hyperactivity-impulsivity, but also with symptoms of disruptive behavior disorders (oppositional-defiant, conduct) and/or anxiety disorders (Avila et al., 2004; Nikolas, Marshall, and Hoelzle, 2019). To date, little is known about how different DSM-5-defined presentations and co-morbid diagnoses influence underlying latent cognitive components of information processing (e.g., Avila et al., 2004; Nikolas, Marshall, and Hoelzle, 2019).

Research using neurocognitive tests found that children with ADHD have slower mean reaction times (RTs) and/or accuracy than those without ADHD across a range of tasks such as those presumed to measure sustained attention (e.g., Baytunca et al., 2018; Huang-Pollock, Karalunas, Tam, \& Moore, 2012; Huang-Pollock et al., 2020; Karalunas et al., 2018; Weigard et al., 2020), inhibitory control (e.g., Baytunca et al., 2018; Fosco et al., 2018; Epstein et al., 2011; Huang-Pollock et al., 2017; Mowinckel, Pedersen, Eilertsen, \& Biele, 2014) or working memory (e.g., Stroux et al., 2016; Kawabe et al., 2018; Nigg et al., 2018). Recently, Ging-Jehli, Ratcliff, and Arnold (2021) reviewed studies across a broad range of neurocognitive tests for ADHD and they found that most studies focused on differences between controls and ADHD and examined specific ADHD populations (e.g., boys only or children without co-morbid diagnoses).

A few studies (e.g., Carr et al., 2010; Collings, 2003; O’Driscoll, 2005; Pritchard, Neumann, \& Rucklidge, 2008) have already examined differences among ADHD presentations. 
For instance, Carr et al. found that children with ADHD-I detected significantly more target letters in a stream of letters than those with ADHD-C though only if they were instructed to divide attention between two types of targets (i.e., type and color of target letters). When having to focus on responding to only the letter type, children with ADHD-I and ADHD-C were equally accurate. O’Driscoll et al. found similar results as Carr et al. in another task (an anti-saccade task in which subjects had to move their eyes either towards the position or towards the opposite position of visually presented target boxes). However, the aforementioned studies focused on summary statistics and on differences between ADHD presentations only, neglecting co-morbid diagnoses.

Research from Cognitive Psychology (e.g., Forstmann, Ratcliff, \& Wagenmakers, 2016; Ratcliff, Huang-Pollock, McKoon, 2018; Ratcliff \& McKoon, 2008) showed that RTs are the product of a decision-making process that consists of multiple cognitive components. Computational models, such as the diffusion decision model (DDM; Ratcliff, 1978), can be used to quantify and separately study each of these cognitive components. Therefore, such a computational modeling approach seems appealing to assess differences in distinct cognitive components among ADHD presentations and between children with different comorbidities.

Understanding similarities and differences in specific cognitive processes among ADHD presentations and between comorbidities is important not only for diagnostic purposes but also for personalized medicine. For instances, studies found that comorbidities can moderate treatment response (Jensen et al, 2001; MTA cooperative group, 1999). One-third of those diagnosed with ADHD are non-responders to the first stimulant tried, and up to $10 \%$ are non-responders to all medication (Adler, Reingold, Morrill, and Wilens, 2006; Swanson et al, 2001; Pliszka, 1989; Arnold, 2000; Arnold et al, 1978). If one identifies and quantifies those cognitive components that 
lead to deficits or biases in information processing, one can target those components in cognitive behavioral therapies.

\section{Current Study}

The purpose of this study was to compare the underlying latent cognitive components between children without ADHD and those with DSM-5 ADHD presentations (analysis 1); and between children with ADHD who had different co-morbid diagnoses, statistically adjusting for differences in DSM-5 ADHD presentations (analysis 2).

We focused on the DSM-5 ADHD presentations "ADHD-I" and "ADHD-C" because research has shown that "ADHD-H" is rare after preschool years (Barkley, 2003; Milich, Balentine, \& Lynam, 2001). Moreover, we focused on studying the underlying latent cognitive components that together resemble information processing by using a specific version of the Continuous Performance Test (CPT) - a common neurocognitive test for ADHD. The latent cognitive components were estimated by applying the DDM to the neurocognitive performance data. In the following sections, we first summarize literature on CPTs for ADHD that are most closely related to this study. Then, we explain the DDM in more detail. We also highlight important differences of this study to previous DDM applications to CPTs in clinical research.

\section{Continuous Performance Tests for ADHD}

The CPT is a commonly used instrument to study cognition in ADHD (Corbett and Constantine, 2006; Epstein et al., 2011; Hervey et al., 2006; Nichols and Waschbusch, 2004). In a CPT, a series of stimuli (e.g., letters, numbers) are presented on a computer screen (or via headphones). Typically, one stimulus represents go trials while another stimulus represents no-go trials. Participants are asked to press a response key for go trials, and to not press the response key for no-go trials. RTs, omission and commission errors serve as performance measures. 
There are two types of CPTs, differing in frequency of go versus no-go trials (see for a review: Edwards et al., 2007). The performance from CPTs with frequent go trials are presumed to elucidate cognitive concepts such as inhibitory control (e.g., Huang-Pollock et al., 2012; but see for critiques also Ging-Jehli et al., 2021). In this type of CPT, children with ADHD often committed more errors (pressing the key when they should not or not pressing the key when they should) than those without ADHD, though evidence remains mixed (Parsons, Duffield, \& Asbee, 2019; Huang-Pollock et al., 2012; Sonuga-Barke, Sergeant, Nigg, \& Willcutt, 2008). Moreover, Collings (2003) found differences among ADHD presentations such that children with ADHD-C conducted significantly more omission errors than those with ADHD-I or those without ADHD. In contrast, other studies failed to find subtype-specific differences (see for a review: Ging-Jehli et al., 2021). The performance from CPTs with rare go trials are presumed to elucidate cognitive concepts such as sustained attention (Robertson et al., 1997; Gordon, 1986; but see for critiques also Ging-Jehli et al., 2021). In this type of CPT, children with ADHD often showed more (omission and commission) errors, slower mean RTs, and greater variability in RTs than those without ADHD (Hervey et al., 2006; Huang-Pollock, Nigg, \& Halperin, 2006; Sergeant, Oosterlaan, van der Meere, 1999). Moreover, Epstein et al. (2011) did not find differences among ADHD presentations, but all children with ADHD had significantly slower mean RTs and lower accuracy than those without ADHD. In contrast, Egeland, Johansen, and Ueland (2009) found that children with ADHD-I had slower mean RTs than those with ADHD-C or without ADHD.

Distinguishing between the two types of CPTs is important because some have argued that ADHD-C is linked to deficits in inhibition control, while ADHD-I is linked to deficits in sustained attention (e.g., Barkley, 1997; Baeyens, 2006; Houghton, Douglas, West, Whiting, Wall, Langsford, \& Caroll, 1999). Therefore, we would expect that CPTs with frequent go trials are 
particular sensitive to the characteristics of ADHD-C while CPTs with rare go trials are particular sensitive to the characteristics of ADHD-I. However, a thorough examination of this hypothesis is lacking thus far because most CPTs involve either frequent go trials (Conners, 2002) or rare go trials (Robertson et al., 1997; Gordon, 1986) but rarely both. Moreover, Ging-Jehli et al. (2021) have recently pointed out that studies are hard to compare because of differences in: i) sample characteristics; ii) definition of ADHD presentations; and iii) task specifics. Therefore, a task that includes both blocks with rare go trials and blocks with frequent go trials might provide information about the cognitive characteristics of ADHD presentations.

A CPT with multiple conditions might be useful for studying the association between latent cognitive components and different ADHD presentations and comorbidities. This is because understanding how children adapt to different task conditions (e.g., transitioning from blocks with frequent go trials to blocks with rare go trials) provides insights into their individual mechanisms of cognitive processing (we provide specific hypotheses in section: Previous DDM Applications To CPTs in ADHD Research). For this study, we used the Integrated Visual and Auditory Continuous Performance Test (IVA-CPT). We refer to the Method section for a detailed description of the IVA-CPT. Compared to the previously discussed CPTs, the IVA-CPT allows for an assessment of both auditory and visual attention on the same task and includes blocks of both frequent and rare go trials (Sandford \& Turner, 2000).

\section{The Diffusion Decision Model (DDM)}

Recent computational modeling applications such as those with the DDM (Ratcliff, 1978) have shown that test performance can be decomposed into underlying cognitive components (e.g., response cautiousness, quality of information integration, response bias) that can be studied separately and that each have established psychological interpretations (Forstmann, Ratcliff, \& 
Wagenmakers, 2016; Huang-Pollock et al., 2017; Wiecki, Poland, \& Frank, 2015). The DDM has also been successfully applied to go/no-go tasks such as CPTs (e.g., Gomez, Ratcliff, Perea, 2007; Huang-Pollock et al., 2017; 2020; Ratcliff, Huang-Pollock, McKoon, 2018).

Pursuing a DDM analysis is interesting because it allows one to understand which cognitive components differ among ADHD presentations and comorbidities. A DDM analysis is more sensitive for detecting cognitive differences between ADHD presentations and comorbidity because it considers the reaction time (RT) distribution (rather than only mean RTs), and simultaneously accounts for correct and error responses from different experimental conditions (Hauser, Fiore, Moutoussis, \& Dolan, 2016; Montague et al. 2012). Therefore, a DDM analysis uses more information than conventional performance measures such as mean RTs or accuracy. The DDM (Ratcliff, 1978) has successfully accounted for the performance in (neuro)cognitive testing from a broad range of clinical populations such as ADHD, autism, depression, anxiety, aphasia (Pe, Vandekerckhove, and Kuppens, 2013; Pirrone, Moustafa et al., 2015; White, Ratcliff, Vasey and McKoon, 2010a, 2010b; Zeguers et al., 2011).

The DDM is based on the most dominant theory of how people make decisions (Forstmann, Ratcliff \& Wagenmakers, 2016). It is presumed that decisions (such as those in laboratory settings) are a result of decision-making processes that have a starting point and that evolve by sequentially accumulating (noisy) evidence up to a specific criterion at which time a response is initiated. A graphical illustration of the model (Supplemental Figure S1), including a description, is provided in the Supplemental Material. In the following paragraphs, we describe the main DDM parameters $\left(a, z / a, T_{e r}, v, d c\right)$ that represent the decomposed cognitive components of the decision-making process. 
Boundary Separation (a). Boundary separation indexes a participant's general tendency towards cautious vs. hasty responses (Ratcliff and McKoon, 2008; Voss, Rothermund, and Voss, 2004). Participants with more cautious response strategies (preferring accurate over fast decisions) have larger boundary separations than participants with less cautious response strategies. Generally, a larger boundary separation results in slower but more accurate responses. Boundary separation varies by the introduction of different instructions (emphasis of speed vs. accuracy; Mulder et al., 2010), reward for accurate responses (Voss et al., 2004), or difficult conditions (Schmitz \& Voss, 2012).

Starting point bias $(z / a)$. Biases in staring points measure a participants' a priori tendency towards go or no-go responses. Previous studies found larger starting point biases $(z / a)$ for ADHD compared to controls; particularly when interstimulus intervals were short rather than long (Huang-Pollock et al., 2016; 2012).

Nondecision Time $\left(\boldsymbol{T}_{e r}\right)$. Nondecision time represents the latency of processes outside the decision process such as task preparation, perceptual encoding of cue, and response execution (i.e., the latency from having reached a decision to pressing the response key associated with that decision). Nondecision time is longer by the introduction of task switches (Ging-Jehli \& Ratcliff, 2020; Schmitz and Voss, 2012), by aging (Cohen-Gilbert et al., 2014; Ratcliff, Thapar, \& McKoon, 2006), and poor individual allocation of attention at the beginning of a trial (Nunez, 2015).

Drift Rate $(v)$. Drift rate represents the quality of integrating stimulus information. Larger drift rates represent faster and more accurate responses, whereas lower drift rates represent slower and less accurate responses. Drift rates are lower in more difficult conditions than in easier conditions (Ratcliff \& McKoon, 2008; Voss et al., 2004). Multiple studies found positive associations of drift rate with IQ and working memory capacity (Ratcliff, Thapar, \& McKoon, 
2010). Previous meta-analyses found lower drift rates for children and adults with ADHD as compared to children and adults without ADHD (Huang-Pollock et al., 2012; Mowinckel, et al., 2015).

Drift Criterion $(\boldsymbol{d c})$. Drift criterion represents the context sensitivity of integrating stimulus information (e.g., Ratcliff \& McKoon, 2008; Smith \& Ratcliff, 2015). For instance, Smith and Ratcliff (2015) explained that changes in drift criterion measure biases in drift rates when stimulus types differ in reward rates or their relative frequency (see also discussions about drift bias: Starns, Ratcliff, \& White, 2012; Kloosterman et al., 2019).

\section{Previous DDM Applications To CPTs In ADHD Research}

Our study allowed us to address several limitations of past DDM applications in the field of ADHD research:

First, previous DDM applications consistently found that children with ADHD have impaired information processing (lower drift rates) than those without ADHD across a range of tasks (see for a detailed review: Ging-Jehli et al., 2021; see also Fosco et al., 2018; Huang-Pollock et al., 2017; 2020; Karalunas et al., 2018; Mowinckel, Pedersen, Eilertsen, \& Biele, 2014; Nigg et al., 2018; Weigard et al., 2020). However, these studies did not account for different DSM-defined presentations and co-morbid diagnoses.

Second, past model applications used neurocognitive tests that involved visual targets only. However, attention to auditory information seems important to many areas of functioning and learning. Most importantly, we predicted that different ADHD presentations show differences in auditory versus visual attention. Processing of auditory and visual information occurs in separate human brain areas (Arnott \& Alain, 2011; Salo, Rinne, Salonen, \& Alho, 2013); and other clinical studies found that ADHD presentations differ in some of these brain areas (Fair et al., 2013; Loo 
et al., 2003). For instance, Fair et al. found that children with ADHD-C had, among others, atypical connectivity in the insular cortex. Functional brain imaging studies showed that the insular cortex is involved in directing particularly auditory attention during cognitive tasks (e.g., Bamiou, Musiek, \& Luxon, 2003). Moreover, the timing and intensity of attention is more important for processing auditory rather than visual information because auditory information is temporally sequenced and of short duration, not allowing "a second look." To address the neglect of auditory data in previous reports, we used a Continuous Performance Test (CPT) that involved both visual and auditory targets.

Third, past DDM applications to CPTs included either blocks with frequent or rare go trials (Ging-Jehli et al., 2021). However, based on the studies previously discussed, it may be that cognitive processing of ADHD-C is particularly sensitive to blocks with frequent go trials, while cognitive processing of ADHD-I is particularly sensitive to blocks with rare go trials. The CPT we used involved both blocks with frequent and rare go trials (targets).

\section{Method}

\section{Participants}

ADHD Sample. 150 children $^{1}$ aged 7 to 10 had the baseline assessment in the International Collaborative ADHD Neurofeedback (ICAN; Neurofeedback Collaborative Group, 2020) randomized clinical trial (NCT02251743). Children were required to: meet DSM-5 ADHD diagnostic criteria for inattentive or combined presentation (APA, 2013) assessed with the Children's Interview for Psychiatric Syndromes (Weller et al., 1999; see Instruments: ChIPS) and doctoral clinician interview; have an IQ $\geq 80$ assessed with the Wechsler Abbreviated Scale of

\footnotetext{
${ }^{1}$ From the 165 participants initially enrolled into the ICAN study, we excluded 14 participants who did not complete the neurocognitive test, and one participant who did not meet the DSM-5 criteria for ADHD.
} 
Intelligence (WASI, Wechsler, 1999), have an electroencephalographic Theta/Beta-ratio (TBR ${ }^{2}$ ) $\geq 4.5$ assessed with the Thought Technology ADHD Suite, and item mean $\geq 1.5$ SD above norms on the Conners- $3^{\text {rd }}$ rating scale (Conners, 2008) by both parent and teacher while off medication. As part of the baseline assessment, children performed the IVA-CPT without medication. (Of the 150 children, 43 were taking medication, which they stopped for 5 days before assessment).

Control Sample. Sixty children without ADHD aged 7 to 10 participated in one visit to perform the IVA-CPT. The controls were required to meet the following inclusion criteria: absence of any DSM-5 psychiatric diagnoses, no head injury with loss of consciousness, and no current psychotherapy or physical or occupational therapy. Children were excluded if they were taking medication for seizures, mood, anxiety, or other DSM-5 disorders. Prospective participants were pre-screened for eligibility via telephone prior to coming to the visit. We additionally used the parent-rated Conners- $3^{\text {rd }}$ edition rating scale to screen for undiagnosed ADHD and symptom severity of inattention and hyperactivity-impulsivity (see Instruments: C-3:P). When analyzing the parents' ratings on the $\mathrm{C}-3: \mathrm{P}$, we noticed that three controls had ratings of total ADHD symptoms above norms. We therefore excluded those three children from all analyses.

\section{Instruments}

Conners-3 ${ }^{\text {rd }}$ edition: Parent Report Long Version (C-3:P; Conners, 2008). The C-3:P was used to assess DSM-IV criteria of ADHD symptoms and symptom severity. All parents completed the C-3:P and were asked to rate the frequency of child behaviors on a 4-point scale $(0=$ not true at all, $1=$ just a little true, $2=$ pretty much true, $3=$ very much true $)$. The questionnaire

\footnotetext{
${ }^{2}$ TBR refers to the ratio between the slow wave theta frequency band $(4$ to $7 \mathrm{~Hz})$ and the fast wave beta frequency band $(13$ to $30 \mathrm{~Hz})$. TBR has been utilized as inclusion criteria for neurofeedback studies because research suggested high TBR to predict good response to neurofeedback (Arns, Drinkenburg, \& Kenemans, 2012; Janssen et al., 2016; Monastra, Monastra, \& George, 2002). $77 \%$ of all prospective children diagnosed with ADHD met the TBR inclusion criterion.
} 
included 108 questions, with 21 items used to measure DSM-IV ADHD symptoms (10 items for inattention symptoms and 11 items for hyperactivity/impulsivity symptoms). We used the T-scores of parent-rated inattention and hyperactivity-impulsivity (both DSM-5 scales) to test for differences between the ADHD sample and controls.

\section{Children's Interview for Psychiatric Syndromes -child (ChIPS) and -parent (P-} ChIPS; Weller et al., 1999). The ChIPS/P-ChIPS is a structured diagnostic interview that was administered at baseline to the children in the ADHD sample to determine DSM-5-defined disorders. Comorbid diagnoses as well as ADHD presentations were taken from the ChIPS/PChIPS, which assessed for 20 DSM-IV psychiatric diagnoses (ADHD, ODD, CD, Substance Abuse, Specific Phobia, Social Phobia, Separation Anxiety Disorder, Generalized Anxiety Disorder, Obsessive-Compulsive Disorder, Stress Disorders [ASD/PTSD], Anorexia, Bulimia, Depression/Dysthymia [MDD/DD], Mania/Hypomania, Enuresis, Encopresis, and Schizophrenia/Psychosis). Diagnoses of co-morbidities were given if either the child or parent endorsed criteria of the disorder. For ADHD, DSM-IV and DSM-5 criteria are identical for this age range except for a more liberal age of onset (before age 12) in DSM-5.

Demographic Questionnaire. Parents of children with and without ADHD completed a demographic questionnaire that included questions such as the child's sex, age, educational setting, overall household income and primary caregiver's education (Table 1). We used this questionnaire to test for socio-demographic differences between the ADHD sample and controls.

Neurocognitive Testing (IVA-CPT $2^{\text {nd }}$ edition; Sandford and Turner, 2002). The Integrated Visual and Auditory Continuous Performance Test (IVA-CPT) is composed of 500 trials with an additional 10 warm-up and cool-down trials, respectively. On each trial, participants are presented with either the number "1" (go trial) or the number "2" (no-go trial). Participants are 
instructed to click the button of a computer mouse when the number " 1 " is presented (either visually or auditorily), but to refrain clicking any buttons when the number "2" is presented (either visually or auditorily). The IVA-CPT involves eight conditions, determined by: two block types (frequent versus rare go trials), two trial types (go versus no-go trials), and two modalities (visual versus auditory trials). In blocks with frequent go trials, $84 \%$ of all trials are go trials. In blocks with rare go trials, $16 \%$ of all trials are go trials (for additional details see Supplemental Material). The IVA-CPT was developed based on the DSM-IV-TR diagnostic criteria for ADHD (APA, 2000). It has made significant contributions to neuropsychological testing for several medical and psychological disorders for different age groups, including adults and children with ADHD (Corbett and Constantine, 2006; Moreno-García, Delgado-Pardo, and Roldán-Blasco, 2015; Park, et al., 2011; Tinius, 2003). However, different studies relied on different outcome measures with varying norms (measures provided by test makers without reporting mean RTs and/or accuracy) and none of these previous IVA studies applied a DDM analysis, thus neglecting the study of underlying cognitive components.

\section{Procedure}

This research was approved by [removed for blinded review] Institutional Review Board (IRB), and written informed consent and assent were obtained from parents and children. Participants were recruited through flyers posted in local community centers and elementary schools. Controls (and at least one of their parents) were invited to a one-time visit (1 hour). Parents completed questionnaires (Method section: Instrument), while their child performed the IVA-CPT on a computer in a separate room (accompanied by a research assistant). Instructions on the IVACPT were standardized and integrated into the computerized test administration. All participants were reimbursed for participation regardless of performance. 


\section{Estimating DDM parameters}

Model Parametrization. The IVA-CPT involves eight conditions (two block types x two trial types $\mathrm{x}$ two modalities) and we introduced: one value for boundary separation ( $a$ ) for all conditions; eight drift rates; one for each trial type (go vs. no-go), modality (visual vs. auditory), and block type (frequent go vs. rare go); and four nondecision times; one for each modality and block type. We provide additional explanation about model parametrization in the Supplemental Material and in the Supplemental Figures S2 and S3. The DDM additionally includes variability parameters (for explanations see Supplemental Material), but we focused our analysis on main parameters. All estimated model parameters can be found in the Supplemental Tables S1 and S2. Model Fitting Procedure. DDM parameter estimates were obtained by using a standard method (Ratcliff, Huang-Pollock, \& McKoon, 2018; Huang-Pollock et al., 2020; Ratcliff \& Tuerlinckx, 2002) in which model parameters were adjusted to obtain a minimum value of chi-square. We explain in detail how the DDM was fit to the data of each participant in the Supplemental Material. Goodness Of Fit. We assessed goodness of fit in different ways (as suggested by Ratcliff et al., 2018; Ratcliff \& Tuerlinckx, 2002), discussed in detail in the Supplemental Material. First, the Supplemental Table S3 shows the mean chi-square goodness of fit values and the degrees of freedom. All the chi-square values were below the critical chi-square values, which suggests that the model fit the data well. Moreover, the fits are quite reasonable compared to previous studies that applied the DDM to other CPTs (e.g., Ratcliff et al., 2018; Gomez et al., 2007). Second, Supplemental Figure S4 illustrates plots of model predictions against data for response proportions (i.e., accuracy) and the .1, .5, and .9 quantile RTs for each condition and for each participant. Supplemental Figure S4 further demonstrates that the model fit the data well. Alternative Model Analyses. We also provide in the Supplemental Material the results of an alternative model (i.e., 
a model with a more flexible model parameterization) which provided poorer fits than the model introduced in the main manuscript.

\section{Statistical Analysis}

We used a DDM analysis to compare the cognitive components between children without ADHD and those with DSM-5 ADHD presentations ${ }^{3}$ (analysis 1); and between children with ADHD and different comorbidities (analysis 2).

We concentrated our analysis on four DDM parameters: $z / a, T_{e r}, v$, and $c_{v}$ (we present all model parameters for each participant group in the Supplemental Material). In the Introduction, we explained that our focus lay on examining how cognitive processing changes across modality (visual vs. auditory) and block type (frequent vs. rare go trials). Therefore, we averaged the drift rates for go trials and no-go trials for each block type and each modality ${ }^{4}$. This resulted in four drift rates and the larger the drift rate, the better (i.e., faster and more accurate) is information integrated when processing the stimulus. Hence, in line with past research discussed in the Introduction, we refer to drift rates as efficiency of the information integration process. In the Introduction, we also explained that changes in drift criteria $(d c)$ refers to biases in drift rates across blocks due to differences in target frequency (e.g., proportion of go versus no-go trials; see: Smith \& Ratcliff, 2015). Therefore, we calculated drift biases by taking the difference between drift rates for no-go trials ${ }^{5}$ and go trials for each block type and for each modality. This resulted into four drift biases $\left(c_{v}\right)$ and the larger the drift bias (i.e., deviation from zero), the more is stimulus

\footnotetext{
${ }^{3}$ For ADHD, DSM-IV and DSM-5 criteria are identical for this age range except for a more liberal age of onset (before age 12) in DSM-5.

${ }^{4}$ As in past studies, drift rates for go trials had a positive sign and drift rates for no-go trials had a negative sign. This is to indicate that each choice (go versus no-go) is represented by a separate decision boundary (see Supplemental Figure S1 and additional explanation in the Supplemental Material). Therefore, we averaged the drift rates for go trials and the absolute values of drift rates for no-go trials.

${ }^{5}$ Absolute values of drift rates for no-go trials (see footnote 4).
} 
processing dependent on the type of trial (go vs. no-go). If drift rates for go trials and no-go trials are approximately equally large, then this suggest good stimulus discrimination irrespective of the stimulus type. Hence, in line with past research discussed in the Introduction, we refer to drift bias as context sensitivity, the sensitivity of the information integration process to stimulus type (go vs. no-go).

For analysis 1. We conducted two separate ANOVAs since different model parameters were kept fixed across different conditions (Method section: Model parametrization). In all ANOVAs, participant group was the between-subject factor. In the first ANOVA, we tested for group-specific differences in starting point bias $(z / a)$. The block type (frequent vs. rare go trials) presented the within-subject factor. In the second mixed ANOVA, we tested for group-specific differences in nondecision time $\left(T_{e r}\right)$, drift rate $(v)$ and drift bias $\left(c_{v}\right)$. The block type and the modality (auditory vs. visual trials) presented the within-subject factors. Since hypothesis tests were repeatedly conducted, respective Bonferroni corrections were incorporated into all the analyses. We also used contrast tests to further examine group-specific differences in main model parameters. We will specify these contrast tests in the forthcoming sections when we introduce the results. For analysis 2 . We included all children with $\operatorname{ADHD}(N=150)$ and assigned them to one of four comorbidity groups based on their CHIPS/CHIPS-P diagnosis: neither, anxiety only, oppositional defiant disorder (ODD) only, or both. Table 1 shows the number of children in each comorbidity group sorted by the DSM-5 presentations ADHD-I and ADHD-C. We accounted for the effects of DSM-5 presentations in the comorbidity analysis (including it as a covariate into our analysis) since the number of DSM-5-defined presentations varied among comorbidity groups. 


\section{Results}

\section{Sample Characteristics}

Sample characteristics are provided in Table 1. Both ADHD groups had significantly higher inattention and hyperactivity-impulsivity T-scores than the controls. The two ADHD groups had similar inattention T-scores, but the ADHD-I group had significantly lower hyperactivity-impulsivity T-scores than the ADHD-C group. The controls included more girls than either ADHD group. ADHD-I and ADHD-C groups did not differ in gender composition. Comorbid diagnoses varied as a function of DSM-5 presentation. Compared to controls, children with ADHD were more likely to attend special classes or a charter school. Moreover, controls were more likely to come from upper income households than children with ADHD. There were no differences in primary caregiver's education between children with ADHD and controls.

\section{Differences between DSM-5-defined ADHD presentations and controls}

We analyzed differences in cognitive components between controls $(N=57)$ and children with ADHD who were sub-grouped into either ADHD-C $(N=99)$ or ADHD-I $(N=51)$ based on DSM-5 presentation. We included sex, child's educational setting, and annual household income as covariates into our analysis due to differences between controls and ADHD groups. Table 2 shows the model parameter values (and corresponding test statistics) for each diagnostic group (controls, ADHD-C, ADHD-I). The Supplemental Figure S3 shows the entire RT distributions for correct and error responses across all conditions and for each of the three diagnostic groups (controls, ADHD-C, ADHD-I). We also provide additional analyses of conventional performance measures (e.g., mean RTs for correct and error responses, omission errors, commission errors) in the Supplemental Table S4. 
Tendency towards premature decisions (z/a). Table 2 shows that the three diagnostic groups did not differ in their tendency toward premature decisions $(z / a)$. All children (controls, ADHD-C, ADHD-I) showed a significantly greater tendency towards premature decisions (z/a: bias towards go-responses) in blocks with frequent go trials than blocks with rare go trials (Table 2).

Deficits in task preparation and response execution $\left(T_{e r}\right)$. Children with either ADHD-C or ADHD-I were slower in task preparation and response execution $\left(T_{e r}\right)$ than controls, but this difference was not statistically significant (Table 2).

Efficiency of processes involved in information integration (v). The controls were significantly more efficient in information integration (larger $v$ ) than children with ADHD-C or ADHD-I (Table 2). Children with ADHD-C were nominally less efficient in information integration (lower $v$ ) than children with ADHD-I, but this difference was not statistically significant (Table 2).

Context sensitivity of processes involved in information integration $\left(c_{v}\right)$. We found a significant diagnostic group x modality interaction (Table 2). Figure 1A illustrates that children with ADHD-C were more sensitive to changes in presentation modality (auditory vs. visual) than children with ADHD-I and controls (i.e., larger changes in $c_{v}$ between auditory and visual trials).

We also found a significant diagnostic group $\mathrm{x}$ modality $\mathrm{x}$ block type interaction (Table 2). Figure 1B and 1C illustrate larger group differences for blocks with frequent go trials (Figure 1B) than for blocks with rare go trials (Figure 1C). For blocks with frequent go trials, we found an interesting relationship: For auditory trials, children with both ADHD-I and ADHD-C had significantly larger $c_{v}$ than controls [control vs. ADHD-C; $t(204)=-2.39, p=0.018$; control vs. ADHD-I; $t(204)=-2.71, p=0.007$; ADHD-C vs. ADHD-I: $t(204)=0.06, p=0.955]$. For visual 
trials, children with ADHD-C had significantly smaller $c_{v}$ than controls and children with ADHDI $[$ ADHD-C vs. control; $t(204)=2.56, p=0.011$; ADHD-C vs. ADHD-I; $t(204)=2.48, p=0.014$; control vs. ADHD-I: $t(204)=-0.15, p=0.884]$. Hence, presenting information visually in a context with frequent targets helped children with ADHD-C in integrating information. In contrast, children with ADHD-I did not show any sensitivity to changes in presentation modality (i.e., equally large $c_{v}$ for auditory and visual trials). Rather, children with ADHD-I seemed to respond predominantly "go" to most trials (i.e., large positive $c_{v}$ indicating a large drift rate for go trials and a low drift rate for no-go trials).

Comparing how $c_{v}$ changed across block types showed another interesting relationship: For blocks with frequent go trials (presumed to tax inhibitory control), processes involved in integrating auditory information were most sensitive, while in blocks with rare go trials (presumed to tax sustained attention), processes involved in integrating visual information were most sensitive.

\section{Differences between comorbidity groups and controls}

We tested for comorbidity-specific cognitive differences among children with ADHD, above and beyond the effects of DSM-5 presentations discussed before (Method section: Statistical Analysis. Analysis 2). Table 4 shows the model parameter values (and corresponding test statistics) for each comorbidity group (neither, anxiety only, ODD only, both). We also provide additional analyses of conventional performance measures (e.g., mean RTs for correct and error responses, omission errors, commission errors) in the Supplemental Table S5.

Tendency toward premature decisions $(z / a)$. Table 4 shows that the four comorbidity groups differed in their tendency toward premature decisions $(z / a$; significant block type $\mathrm{x}$ comorbidity group interaction). Figure 2 illustrates that all four comorbidity groups showed 
increased tendency toward premature decisions ( $z / a$ : bias towards go-responses) in blocks with frequent go trials compared to blocks with rare go trials. However, the ADHD group with both comorbid anxiety and ODD (i.e., both group) had a significantly larger tendency toward premature decisions than the other three comorbidity groups (Table 4).

Deficits in task preparation and response execution $\left(T_{e r}\right)$. Table 4 illustrates that the four comorbidity groups were similarly fast in task preparation and response execution $\left(T_{e r}\right)$.

Efficiency $(v)$ and context sensitivity $\left(c_{v}\right)$ of processes involved in information integration.

The four comorbidity groups showed similar efficiency in information integration $(v)$ as well as context sensitivity information integration to go vs. no-go stimuli $\left(c_{v}\right)$.

\section{Discussion}

This is the first study to examine clinical associations of distinct cognitive components with DSM-5-defined ADHD presentations (analysis 1) and comorbidity groups (analysis 2) using a computational modeling approach (i.e., DDM).

Our results suggest important differences in specific cognitive components among ADHD presentations and between children with different comorbidities. These findings provide insights regarding heterogeneity of cognitive characteristics among children with ADHD. Specifically, examining differences between controls and ADHD presentations, we found that controls had, on average, a significantly better quality of information integration (larger $v$ ) than children with ADHD-C or -I. This means that controls had faster and more accurate processing than those with ADHD for both visual and auditory stimuli. Previous studies that used other CPTs (with visual trials only) and that focused on children with ADHD-C are consistent with our finding (HuangPollock et al., 2016; 2012; Mowinckel, et al., 2015). 
We extend past research by using the IVA-CPT (with both auditory and visual trials) and finding a significant diagnostic group x modality interaction. Specifically, children with ADHD$\mathrm{C}$ were more sensitive than those with ADHD-I and controls to whether stimuli were auditory or visual (Figure 1A: larger changes in $c_{v}$ ). These results could be important for educational strategies regarding the most useful modality for presentation of educational materials: in a context with frequent targets (go stimuli), presenting them visually rather than auditorily helped particularly children with ADHD-C to achieve faster and more accurate processing. One may speculate that a context with frequent target stimuli is more engaging, but also more demanding, and presenting material visually rather than auditorily helped children with ADHD-C to integrate information better (perhaps by allowing them to "take a second look"). However, further studies are needed to assess to which extent these results generalize.

In a context with rare target stimuli, presenting information auditorily rather than visually helped children with both ADHD-C and ADHD-I to achieve faster and more accurate processing. This conclusion is evidenced by smaller drift biases (decreased sensitivity of information integration to go vs. no-go stimuli, suggesting robust information integration) for auditory than visual trials and similar drift rates (efficiency of information integration) for auditory and visual trials. One may speculate that a context with rare target stimuli is more boring and presenting material auditorily rather than visually helped children with ADHD to stay on the task (with all $v$ being higher compared to those of the blocks with frequent go trials). Conversely, perhaps the rarity of go stimuli in the blocks with rare go trials makes the go stimuli "stick out" and appear novel/interesting. However, further studies are needed to assess to what extent these results generalize. 
A few studies examined the cognitive characteristics of DSM-defined ADHD presentations in other CPTs (with fewer conditions than the IVA-CPT), but without a DDM analysis. Results remained mixed (e.g., Collings, 2003; Epstein et al., 2011). For instance, Collings (2003) administered a CPT to children with ADHD-C, ADHD-I, and controls. He found that ADHD-C made significantly more omission errors than ADHD-I and controls. In contrast to the results found by Collings (2003), Epstein et al. (2011) did not find significant differences between DSM-defined ADHD presentations; neither in the number of omission and commission errors nor in the mean RTs. Both studies relied on a CPT with only visual trials and with only blocks of rare go trials. We also did not find any significant differences between DSM-defined ADHD presentations for blocks with rare go trials; a result further consistent with Lin et al. (2017)'s findings discussed in the Introduction. However, for blocks with frequent go trials, we found that DSM-defined ADHD presentations significantly differed in their ability to respond to auditory relative to visual trials (as reflected by the previously discussed finding that ADHD-C, more so than ADHD-I, processed auditory stimuli slower and less accurately than visual stimuli). Our findings highlight the importance of cognitive tests with multiple conditions because our results show that clinical associations appear when we examine how cognitive components change across conditions.

Accounting for different co-morbid disorders (above and beyond DSM-5 ADHD presentations), we found that doubly comorbid children with ADHD (with both ODD and anxiety disorders) showed a significantly increased tendency towards premature responses (larger $z / a$ ) for blocks with frequent go trials (relative to blocks with rare go trials) than children with ADHD and anxiety only, ODD only, or no co-morbid disorder. This finding highlights the confounding effect of "comorbidity load," suggesting additional biases in cognitive processing with double comorbidity. One may speculate that the larger shifts in starting point bias $(z / a)$ due to transitions 
between block types in children with ADHD and double comorbidity highlights their tendency to overly adjust prior expectations (about what follows next) in response to contextual changes. Overly adjusting expectations can be a blessing in some contexts, but a curse in other contexts, and raising awareness of such biases in an individual's decision-making process could be an important target of CBT. Specifically, our results suggest that treatments are needed that help children with both ODD and anxiety disorders to overcome response biases, avoiding making premature conclusions. One might speculate that a synergism of anxiety and oppositional-defiant tendencies could lead to a cognitive stance like "I expect them to try to fool me, but I'm on guard and will outsmart them by adjusting my responses when they try to trap me with a change of rules."

Strengths of this study include the large sample of children with ADHD and the application of a cognitive task that involves multiple conditions. However, limitations include the administration of only one cognitive task. Future studies could administer a battery of cognitive tasks and apply a hierarchical DDM analysis to those tasks to examine the generalizability of DDM results to other tasks. Moreover, the clinical sample in this study did not include other frequent comorbidities associated with ADHD, such as autism spectrum disorder, and thus is not representative of the whole child mental health clinical population. Some of our findings are restricted to children who had a diagnosis of ADHD and who had an electroencephalographic TBR of at least 4.5 (see for details Method section: Participants). $77 \%$ of all prospective participants Meeting DSM-5 ADHD critieria met the TBR inclusion criterion. While our results provide insights into the cognitive characteristics of different DSM-5 defined presentations and comorbidities, the question remains how fine-grained a subgroup analysis should be. The more specific and smaller the subgroups, the larger the risk that results are not generalizable. In this study, the children with ADHD had co-morbid anxiety disorders and/or oppositional defiant 
disorders which led to a relatively small subgrouping (i.e., 4 comorbidity groups: ADHD+anxiety, ADHD+ODD, ADHD+anxiety+ODD, or ADHD-only). In a sample with a larger variety of comorbid diagnoses, one sensitive approach could be to group comorbid diagnoses into externalizing and internalizing disorders. Finally, we focused our analysis on differences between DSM-defined presentations and co-morbid diagnoses. Future studies should also focus on a dimensional perspective of ADHD by examining how DDM model parameters relate to symptom severity of inattention and hyperactivity-impulsivity (see for further discussions: Ging-Jehli et al., 2021; Salum et al. 2014).

\section{Conclusion}

Our results suggest that neurocognitive testing, in conjunction with computational models, can be used to index different cognitive patterns distinguishing ADHD presentations from controls and from each other and distinguishing comorbidity effects. Moreover, we showed that a CPT with multiple conditions, in conjunction with a computational modeling analysis, adds important insights by helping to characterize and quantify biases in distinct cognitive components. Identification of the underlying latent cognitive components of different ADHD presentations and co-morbid diagnoses could help to select and design treatments tailored to the needs of different individuals with ADHD. 


\section{References}

Adler, L. A., Reingold, L. S., Morrill, M. S., and Wilens, T. E. (2006). Combination pharmacotherapy for adult ADHD. Current psychiatry reports, 8(5), 409-415. doi: 10.1007/s11920-006-0044-9

American Psychiatric Association. (2000). Diagnostic and statistical manual of mental disorders (4th ed.). Washington, DC: American Psychiatric Association.

American Psychiatric Association (2013). Diagnostic and Statistical Manual of Mental Disorders, fifth edition. American Psychiatric Publishing, Arlington, VA.

Arnold, L. E. (2000). Methyiphenidate vs. amphetamine: Comparative review. Journal of Attention Disorders, 3(4), 200-211. doi:10.1177/108705470000300403

Arnold, L. E., Christopher, J., Huestis, R., \& Smeltzer, D. J. (1978). Methylphenidate vs dextroamphetamine vs caffeine in minimal brain dysfunction: controlled comparison by placebo washout design with Bayes' analysis. Archives of General Psychiatry, 35(4), 463473.

Arns M, Drinkenburg W, and Kenemans JL (2012). The Effects of QEEG-Informed Neurofeedback in ADHD: An Open-Label Pilot Study. Appl Psychophysiol Biofeedback. 37(3):171-80. doi:10.1007/s10484-012-9191-4.

Arnott, S. R., \& Alain, C. (2011). The auditory dorsal pathway: Orienting vision. Neuroscience \& Biobehavioral Reviews, 35(10), 2162-2173. doi:10.1016/j.neubiorev.2011.04.005

Avila, C., Cuenca, I., Félix, V., Parcet, M., \& Miranda, A. (2004). Measuring impulsivity in school-aged boys and examining its relationship with ADHD and ODD ratings. Journal of Abnormal Child Psychology, 32, 295 -304. doi:10.1023/B:

JACP.0000026143.70832.4b.

Baeyens, D., Roeyers, H., \& Walle, J. V. (2006). Subtypes of attention-deficit/hyperactivity disorder (ADHD): distinct or related disorders across measurement levels?. Child Psychiatry and Human Development, 36(4), 403-417.

Barkley, R. A. (2003). Issues in the diagnosis of attention-deficit/hyperactivity disorder in children. Brain and Development, 25(2), 77-83.

Barkley, R. A. (1997). Behavioral inhibition, sustained attention, and executive functions: constructing a unifying theory of ADHD. Psychological bulletin, 121(1), 65.

Bamiou, D. E., Musiek, F. E., \& Luxon, L. M. (2003). The insula (Island of Reil) and its role in auditory processing: literature review. Brain research reviews, 42(2), 143-154.

Baytunca, M. B., Inci, S. B., Ipci, M., Kardas, B., Bolat, G. U., \& Ercan, E. S. (2018). The neurocognitive nature of children with ADHD comorbid sluggish cognitive tempo: Might SCT be a disorder of vigilance?. Psychiatry research, 270, 967-973. doi: 10.1016/j.psychres.2018.03.038

Carr, L., Henderson, J., \& Nigg, J. T. (2010). Cognitive control and attentional selection in adolescents with ADHD versus ADD. Journal of Clinical Child \& Adolescent Psychology, 39(6), 726-740. doi: 10.1080/15374416.2010.517168

Clark, L. A., Watson, D., \& Reynolds, S. (1995). Diagnosis and Classification of Psychopathology: Challenges to the Current System and Future Directions. Annual Review of Psychology, 46(1), 121-153. doi:10.1146/annurev.ps.46.020195.001005

Coghill, D., \& Sonuga-Barke, E. J. S. (2012). Annual Research Review: Categories versus dimensions in the classification and conceptualisation of child and adolescent mental disorders - implications of recent empirical study. Journal of Child Psychology and Psychiatry, 53(5), 469-489. doi:10.1111/j.1469-7610.2011.02511.x 
Cohen-Gilbert, J. E., Killgore, W. D. S., White, C. N., Schwab, Z. J., Crowley, D. J., Covell, M. J., ... Silveri, M. M. (2014). Differential influence of safe versus threatening facial expressions on decision-making during an inhibitory control task in adolescence and adulthood. Developmental Science, 17(2), 212-223. doi:10.1111/desc.12123

Collings, R. D. (2003). Differences between ADHD inattentive and combined types on the CPT. Journal of Psychopathology and Behavioral Assessment, 25(3), 177-189. doi: 10.1023/a:1023525007441

Conners, C. K. (2002). Conners' continuous performance test (CPTII). Technical guide and software manual. North Tonawanda, NY: Multi Health Systems.

Conners, K. C. (2008). Conners 3rd edition manual. New York: Multi-Health Systems, Inc.

Corbett, B. A., \& Constantine, L. J. (2006). Autism and attention deficit hyperactivity disorder: assessing attention and response control with the integrated visual and auditory continuous performance test. Child Neuropsychology, 12(4-5), 335-348. doi:10.1080/ 09297040500350938

Edwards, M. C., Gardner, E. S., Chelonis, J. J., Schulz, E. G., Flake, R. A., \& Diaz, P. F. (2007). Estimates of the Validity and Utility of the Conners' Continuous Performance Test in the Assessment of Inattentive and/or Hyperactive-Impulsive Behaviors in Children. Journal of Abnormal Child Psychology, 35(3), 393-404. doi:10.1007/s10802-007-9098-3

Egeland, J., Johansen, S. N., \& Ueland, T. (2009). Differentiating between ADHD subtypes on CCPT measures of sustained attention and vigilance. Scandinavian Journal of Psychology, 50(4), 347-354.

Epstein, J. N., Langberg, J. M., Rosen, P. J., Graham, A., Narad, M. E., Antonini, T. N., ... \& Altaye, M. (2011). Evidence for higher reaction time variability for children with ADHD on a range of cognitive tasks including reward and event rate manipulations. Neuropsychology, 25(4), 427-441. doi: 10.1037/a0022155

Fair, D., Nigg, J. T., Iyer, S., Bathula, D., Mills, K. L., Dosenbach, N. U., ... \& Buitelaar, J. K. (2013). Distinct neural signatures detected for ADHD subtypes after controlling for micro-movements in resting state functional connectivity MRI data. Frontiers in systems neuroscience, 6, 80. doi: 10.3389/fnsys.2012.00080

Forstmann, B. U., Ratcliff, R., \& Wagenmakers, E. J. (2016). Sequential sampling models in cognitive neuroscience: Advantages, applications, and extensions. Annual review of psychology, 67(1), 641-666. doi:10.1146/annurev-psych-122414-033645

Fosco, W. D., Kofler, M. J., Alderson, R. M., Tarle, S. J., Raiker, J. S., \& Sarver, D. E. (2018). Inhibitory Control and Information Processing in ADHD: Comparing the Dual Task and Performance Adjustment Hypotheses. Journal of Abnormal Child Psychology, 47(6), 961-974. doi:10.1007/s10802-018-0504-9

Ging-Jehli, N. R., \& Ratcliff, R. (2020). Effects of aging in a task-switch paradigm with the diffusion decision model. Psychology and Aging, 35(6), 850.

Ging-Jehli, N. R., Ratcliff, R., \& Arnold, L. E. (2021). Improving neurocognitive testing using computational psychiatry-A systematic review for ADHD. Psychological Bulletin, 147(2), 169.

Gomez, P., Ratcliff, R., \& Perea, M. (2007). A model of the go/no-go task. Journal of Experimental Psychology: General, 136(3), 389-413. doi:10.1037/0096-3445.136.3.389

Gordon, M. (1986). Instruction manual for the Gordon diagnostic system. DeWitt, NY: Gordon Diagnostic Systems. 
Hauser, T. U., Fiore, V. G., Moutoussis, M., \& Dolan, R. J. (2016). Computational psychiatry of ADHD: neural gain impairments across marrian levels of analysis. Trends in Neurosciences, 39(2), 63-73. doi:10.1016/j.tins.2015.12.009

Hervey, A. S., Epstein, J. N., Curry, J. F., Tonev, S., Eugene Arnold, L., Keith Conners, C., ... Hechtman, L. (2006). Reaction time distribution analysis of neuropsychological performance in an ADHD sample. Child Neuropsychology, 12(2), 125-140. doi:10.1080/09297040500499081

Houghton, S., Douglas, G., West, J., Whiting, K., Wall, M., Langsford, S., ... \& Carroll, A. (1999). Differential patterns of executive function in children with attention-deficit hyperactivity disorder according to gender and subtype. Journal of child neurology, 14(12), 801-805.

Huang-Pollock, C. L., Karalunas, S. L., Tam, H., \& Moore, A. N. (2012). Evaluating vigilance deficits in ADHD: A meta-analysis of CPT performance. Journal of abnormal psychology, 121(2), 360-371. doi: 10.1037/a0027205

Huang-Pollock, C. L., Nigg, J. T., \& Halperin, J. M. (2006). Single dissociation findings of ADHD deficits in vigilance but not anterior or posterior attention systems. Neuropsychology, 20(4), 420-429. doi: 10.1037/0894-4105.20.4.420

Huang-Pollock, C., Ratcliff, R., McKoon, G., Roule, A., Warner, T., Feldman, J., \& Wise, S. (2020). A diffusion model analysis of sustained attention in children with attention deficit hyperactivity disorder. Neuropsychology, 34(6), 641-653. doi:10.1037/neu0000636

Huang-Pollock, C., Ratcliff, R., McKoon, G., Shapiro, Z., Weigard, A., \& Galloway-Long, H. (2017). Using the diffusion model to explain cognitive deficits in attention deficit hyperactivity disorder. Journal of abnormal child psychology, 45(1), 57-68. doi:10.1007/s10802-016-0151-y

Janssen TWP, Bink M, Geladé K, van Mourik R, Maras A, and Oosterlaan J. (2016). A randomized controlled trial into the effects of neurofeedback, methylphenidate, and physical activity on EEG power spectra in children with ADHD. J Child Psychol Psychiatry. 57(5):63344. doi:10.1111/jcpp.12517.

Jensen, P. S., Hinshaw, S. P., Kraemer, H. C., Lenora, N., Newcorn, J. H., Abikoff, H. B., ... \&

Elliott, G. R. (2001). ADHD comorbidity findings from the MTA study: comparing comorbid subgroups. Journal of the American Academy of Child \& Adolescent Psychiatry, 40(2), 147-158. doi:10.1097/00004583-200102000-00009

Karalunas, S. L., Hawkey, E., Gustafsson, H., Miller, M., Langhorst, M., Cordova, M., ... Nigg, J. T. (2018). Overlapping and Distinct Cognitive Impairments in AttentionDeficit/Hyperactivity and Autism Spectrum Disorder without Intellectual Disability. Journal of Abnormal Child Psychology, 46(8), 1705-1716. doi:10.1007/s10802-0170394-2

Kawabe, K., Horiuchi, F., Kondo, S., Matsumoto, M., Seo, K., Oka, Y., \& Ueno, S. I. (2018). Neurocognitive assessment of children with neurodevelopmental disorders: Preliminary findings. Pediatrics International, 60(9), 820-827. doi:10.1111/ped.13662

Killeen, P. R. (2019). Models of Attention-Deficit Hyperactivity Disorder. Behavioural processes, 162, 205-214. doi: 10.1016/j.beproc.2019.01.001

Kloosterman, N. A., de Gee, J. W., Werkle-Bergner, M., Lindenberger, U., Garrett, D. D., \& Fahrenfort, J. J. (2019). Humans strategically shift decision bias by flexibly adjusting sensory evidence accumulation. Elife, 8, e37321. 
Kofler, M. J., Sarver, D. E., Spiegel, J. A., Day, T. N., Harmon, S. L., \& Wells, E. L. (2017). Heterogeneity in ADHD: Neurocognitive predictors of peer, family, and academic functioning. Child Neuropsychology, 23(6), 733-759. doi: 10.1080/09297049.2016.1205010

Lin, H. Y., Hsieh, H. C., Lee, P., Hong, F. Y., Chang, W. D., \& Liu, K. C. (2017). Auditory and visual attention performance in children with ADHD: The attentional deficiency of ADHD is modality specific. Journal of Attention Disorders, 21(10), 856-864.

Loo, S. K., Specter, E., Smolen, A., Hopfer, C., Teale, P. D., \& Reite, M. L. (2003). Functional Effects of the DAT1 Polymorphism on EEG Measures in ADHD. Journal of the American Academy of Child \& Adolescent Psychiatry, 42(8), 986-993. doi:10.1097/01.chi.0000046890.27264.88

McBurnett, K., Villodas, M., Burns, G., Hinshaw, S., Beaulieu, A., \& Pfiffner, L. (2014). Structure and Validity of Sluggish Cognitive Tempo Using an Expanded Item Pool in Children with Attention-Deficit/Hyperactivity Disorder. Journal of Abnormal Child Psychology, 42(1), 37-48. doi: 10.1007/s10802-013-9801-5.

Milich, R., Balentine, A. C., \& Lynam, D. R. (2001). ADHD combined type and ADHD predominantly inattentive type are distinct and unrelated disorders. Clinical Psychology: Science and Practice, 8, 463-488.

Monastra VJ, Monastra DM, and George S. (2002). The effects of stimulant therapy, EEG biofeedback, and parenting style on the primary symptoms of attentiondeficit/hyperactivity disorder. Appl Psychophysiol Biofeedback. 27(4):231-49.

Montague, P. R., Dolan, R. J., Friston, K. J., \& Dayan, P. (2012). Computational psychiatry. Trends in Cognitive Sciences, 16(1), 72-80. doi:10.1016/j.tics.2011.11.018

Moreno-García, I., Delgado-Pardo, G., \& Roldán-Blasco, C. (2015). Attention and response control in ADHD. Evaluation through integrated visual and auditory continuous performance test. The Spanish Journal of Psychology, 18. doi:10.1017/sjp.2015.2

Mowinckel, A. M., Pedersen, M. L., Eilertsen, E., \& Biele, G. (2015). A meta-analysis of decision-making and attention in adults with ADHD. Journal of attention disorders, 19(5), 355-367. doi: 10.1177/1087054714558872

The Collaborative Neurofeedback Group (2020). Double-Blind Placebo-Controlled Randomized

Clinical Trial of Neurofeedback for Attention-Deficit/Hyperactivity Disorder With 13 Month Follow-up. Journal of the American Academy of Child \& Adolescent Psychiatry.

The MTA Cooperative Group (1999). Moderators and Mediators of Treatment Response for

Children With Attention-Deficit/Hyperactivity Disorder. Archives of General Psychiatry,

56(12), 1088. doi:10.1001/archpsyc.56.12.1088

Mulder, M. J., Bos, D., Weusten, J. M., van Belle, J., van Dijk, S. C., Simen, P., ... \& Durston, S. (2010). Basic impairments in regulating the speed-accuracy tradeoff predict symptoms of attention-deficit/hyperactivity disorder. Biological psychiatry, 68(12), 1114-1119. doi: 10.1016/j.biopsych.2010.07.031

Nichols, S. L., \& Waschbusch, D. A. (2004). A review of the validity of laboratory cognitive tasks used to assess symptoms of ADHD. Child Psychiatry and Human Development, 34(4), 297-315. doi: 10.1023/b:chud.0000020681.06865.97

Nigg, J. T. (2001). Is ADHD a disinhibitory disorder?. Psychological bulletin, 127(5), 571-598. doi : 10.1037/0033-2909.127.5.571

Nigg, J. T., Gustafsson, H. C., Karalunas, S. L., Ryabinin, P., McWeeney, S. K., Faraone, S. V., ... \& Wilmot, B. (2018). Working memory and vigilance as multivariate endophenotypes 
related to common genetic risk for attention-deficit/hyperactivity disorder. Journal of the American Academy of Child \& Adolescent Psychiatry, 57(3), 175-182.

Nigg, J. T., Willcutt, E. G., Doyle, A. E., \& Sonuga-Barke, E. J. (2005). Causal heterogeneity in attention-deficit/hyperactivity disorder: do we need neuropsychologically impaired subtypes?. Biological psychiatry, 57(11), 1224-1230. doi:

10.1016/j.biopsych.2004.08.025

Nikolas, M. A., Marshall, P., \& Hoelzle, J. B. (2019). The role of neurocognitive tests in the assessment of adult attention-deficit/hyperactivity disorder. Psychological assessment, 31(5), 685-698. https://doi.org/10.1037/pas0000688

Nunez, M. D. (2015). Individual differences in attention influence perceptual decision making. Frontiers in Psychology, 6. doi:10.3389/fpsyg.2015.00018

O’Driscoll, G. A., Dépatie, L., Holahan, A. L. V., Savion-Lemieux, T., Barr, R. G., Jolicoeur, C., \& Douglas, V. I. (2005). Executive functions and methylphenidate response in subtypes of attention-deficit/hyperactivity disorder. Biological psychiatry, 57(11), 1452-1460. doi:10.1016/j.biopsych.2005.02.029

Parsons, T. D., Duffield, T., \& Asbee, J. (2019). A Comparison of Virtual Reality Classroom Continuous Performance Tests to Traditional Continuous Performance Tests in Delineating ADHD: a Meta-Analysis. Neuropsychology review, 1-19. doi:10.1007/s11065-019-09407-6

Park, M.-H., Kweon, Y. S., Lee, S. J., Park, E.-J., Lee, C., \& Lee, C.-U. (2011). Differences in performance of ADHD children on a visual and auditory continuous performance test according to IQ. Psychiatry Investigation, 8(3), 227. doi:10.4306/pi.2011.8.3.227

Pe, M. L., Vandekerckhove, J., \& Kuppens, P. (2013). A diffusion model account of the relationship between the emotional flanker task and rumination and depression. Emotion, 13(4), 739-747. doi:10.1037/a0031628

Pirrone, A., Johnson, I., Stafford, T., \& Milne, E. (2018). A diffusion model decomposition of orientation discrimination in children with Autism Spectrum Disorder (ASD). European Journal of Developmental Psychology, 17(2), 213-230. doi:10.1080/17405629.2018.1561364

Pliszka, S. R. (1989). Effect of anxiety on cognition, behavior, and stimulant response in ADHD. Journal of the American Academy of Child and Adolescent Psychiatry, 28(6), 882-887. doi:10.1097/00004583-198911000-00012

Pritchard, V. E., Neumann, E., \& Rucklidge, J. J. (2008). Selective attention and inhibitory deficits in ADHD: Does subtype or comorbidity modulate negative priming effects?. Brain and Cognition, 67(3), 324-339. doi:10.1016/j.bandc.2008.02.002

Ratcliff, R. (1978). A theory of memory retrieval. Psychological Review, 85(2), 59-108. doi:10.1037/0033-295x.85.2.59

Ratcliff, R., Huang-Pollock, C., \& McKoon, G. (2018). Modeling individual differences in the go/no-go task with a diffusion model. Decision, 5(1), 42-62. doi:10.1037/dec0000065

Ratcliff, R., \& McKoon, G. (2008). The Diffusion Decision Model: theory and data for two-choice decision tasks. Neural Computation, 20(4), 873-922. doi:10.1162/neco.2008.12-06-420

Ratcliff, R., Thapar, A., \& McKoon, G. (2006). Aging and individual differences in rapid twochoice decisions. Psychonomic Bulletin \& Review, 13(4), 626-635. doi:10.3758/bf03193973

Ratcliff, R., Thapar, A., \& McKoon, G. (2010). Individual differences, aging, and IQ in twochoice tasks. Cognitive psychology, 60(3), 127-157. doi:10.1016/j.cogpsych.2009.09.001 
Ratcliff, R., \& Tuerlinckx, F. (2002). Estimating parameters of the diffusion model: Approaches to dealing with contaminant reaction times and parameter variability. Psychonomic Bulletin \& Review, 9(3), 438-481. doi:10.3758/bf03196302

Ratcliff, R., Van Zandt, T., \& McKoon, G. (1999). Connectionist and diffusion models of reaction time. Psychological Review, 106(2), 261-300. doi:10.1037/0033-295x.106.2.261

Robertson, I. H., Manly, T., Andrade, J., Baddeley, B. T., \& Yiend, J. (1997). “Oops!”: Performance correlates of everyday attentional failures in traumatic brain injured and normal subjects. Neuropsychologia, 35(6), 747-758. https://doi.org/10.1016/s00283932(97)00015-8

Salo, E., Rinne, T., Salonen, O., \& Alho, K. (2013). Brain activity during auditory and visual phonological, spatial and simple discrimination tasks. Brain Research, 1496, 55-69. doi:10.1016/j.brainres.2012.12.013

Salum, G. A., Sonuga-Barke, E., Sergeant, J., Vandekerckhove, J., Gadelha, A., Moriyama, T. S., ... \& Rohde, L. A. P. (2014). Mechanisms underpinning inattention and hyperactivity: neurocognitive support for ADHD dimensionality. Psychological medicine, 44(15), 3189-3201. doi:10.1017/s0033291714000919

Sandford J. A., \& Turner A. (2000). Integrated visual and auditory continuous performance test manual. Richmond, VA: Brain Train.

Schmitz, F., \& Voss, A. (2012). Decomposing task-switching costs with the diffusion model. Journal of Experimental Psychology: Human Perception and Performance, 38(1), 222250. doi:10.1037/a0026003

Sergeant, J. A., Oosterlaan, J., \& van der Meere, J. (1999). Information processing and energetic factors in attention-deficit/hyperactivity disorder. In H. C. Quay \& A. E. Hogan (Eds.), Handbook of disruptive behavior disorders (pp. 1975-104). Dordrecht, Netherlands: Kluwer Academic Publishers. doi:10.1007/978-1-4615-4881-2_4

Smith, P. L., \& Ratcliff, R. (2015). An introduction to the diffusion model of decision making. In An introduction to model-based cognitive neuroscience (pp. 49-70). Springer, New York, NY.

Sonuga-Barke, E. J., Sergeant, J. A., Nigg, J., \& Willcutt, E. (2008). Executive dysfunction and delay aversion in attention deficit hyperactivity disorder: Nosologic and diagnostic implications. Child and Adolescent Psychiatric Clinics of North America, 17(2), 367384. doi:10.1016/j.chc.2007.11.008

Starns, J. J., Ratcliff, R., \& White, C. N. (2012). Diffusion model drift rates can be influenced by decision processes: an analysis of the strength-based mirror effect. Journal of Experimental Psychology: Learning, Memory, and Cognition, 38(5), 1137.

Stroux, D., Shushakova, A., Geburek-Höfer, A. J., Ohrmann, P., Rist, F., \& Pedersen, A. (2016). Deficient interference control during working memory updating in adults with ADHD: an event-related potential study. Clinical Neurophysiology, 127(1), 452-463. doi:10.1016/j.clinph.2015.05.021

Swanson, J. M., Kraemer, H. C., Hinshaw, S. P., Arnold, L. E., Conners, C. K., Abikoff, H. B., ... \& Hechtman, L. (2001). Clinical relevance of the primary findings of the MTA: success rates based on severity of ADHD and ODD symptoms at the end of treatment. Journal of the American Academy of Child \& Adolescent Psychiatry, 40(2), 168-179. doi:10.1097/00004583-200102000-00011 
Tinius, T. (2003). The Integrated Visual and Auditory Continuous Performance Test as a neuropsychological measure. Archives of Clinical Neuropsychology, 18(5), 439-454. doi:10.1016/s0887-6177(02)00144-0

Voss, A., Rothermund, K., \& Voss, J. (2004). Interpreting the parameters of the diffusion model: An empirical validation. Memory \& Cognition, 32(7), 1206-1220. doi:10.3758/bf03196893

Wechsler, D. (1999). Wechsler Abbreviated Scale of Intelligence (WASI) Manual. San Antonio, TX: The Psychological Corporation

Weigard, A., Soules, M., Ferris, B., Zucker, R. A., Sripada, C., \& Heitzeg, M. (2020). Cognitive modeling informs interpretation of go/no-go task-related neural activations and their links to externalizing psychopathology. Biological Psychiatry: Cognitive Neuroscience and Neuroimaging, 5(5), 530-541.

Weller EB, Weller RA, Fristad AF, Rooney MT (1999), Administration Manualfor the ChIPS. Washington, DC: American Psychiatric Press

White, C. N., Ratcliff, R., Vasey, M. W., \& McKoon, G. (2010a). Using diffusion models to understand clinical disorders. Journal of Mathematical Psychology, 54(1), 39-52. doi:10.1016/j.jmp.2010.01.004

White, C. N., Ratcliff, R., Vasey, M. W., \& McKoon, G. (2010b). Anxiety enhances threat processing without competition among multiple inputs: A diffusion model analysis. Emotion, 10(5), 662-677. doi:10.1037/a0019474

Wiecki, T. V., Poland, J., \& Frank, M. J. (2015). Model-based cognitive neuroscience approaches to computational psychiatry: clustering and classification. Clinical Psychological Science, 3(3), 378-399. doi:10.1177/2167702614565359

Zeguers, M. H. T., Snellings, P., Tijms, J., Weeda, W. D., Tamboer, P., Bexkens, A., \& Huizenga, H. M. (2011). Specifying theories of developmental dyslexia: a diffusion model analysis of word recognition. Developmental Science, 14(6), 1340-1354. doi:10.1111/j.14677687.2011.01091.x 


\section{Figures}

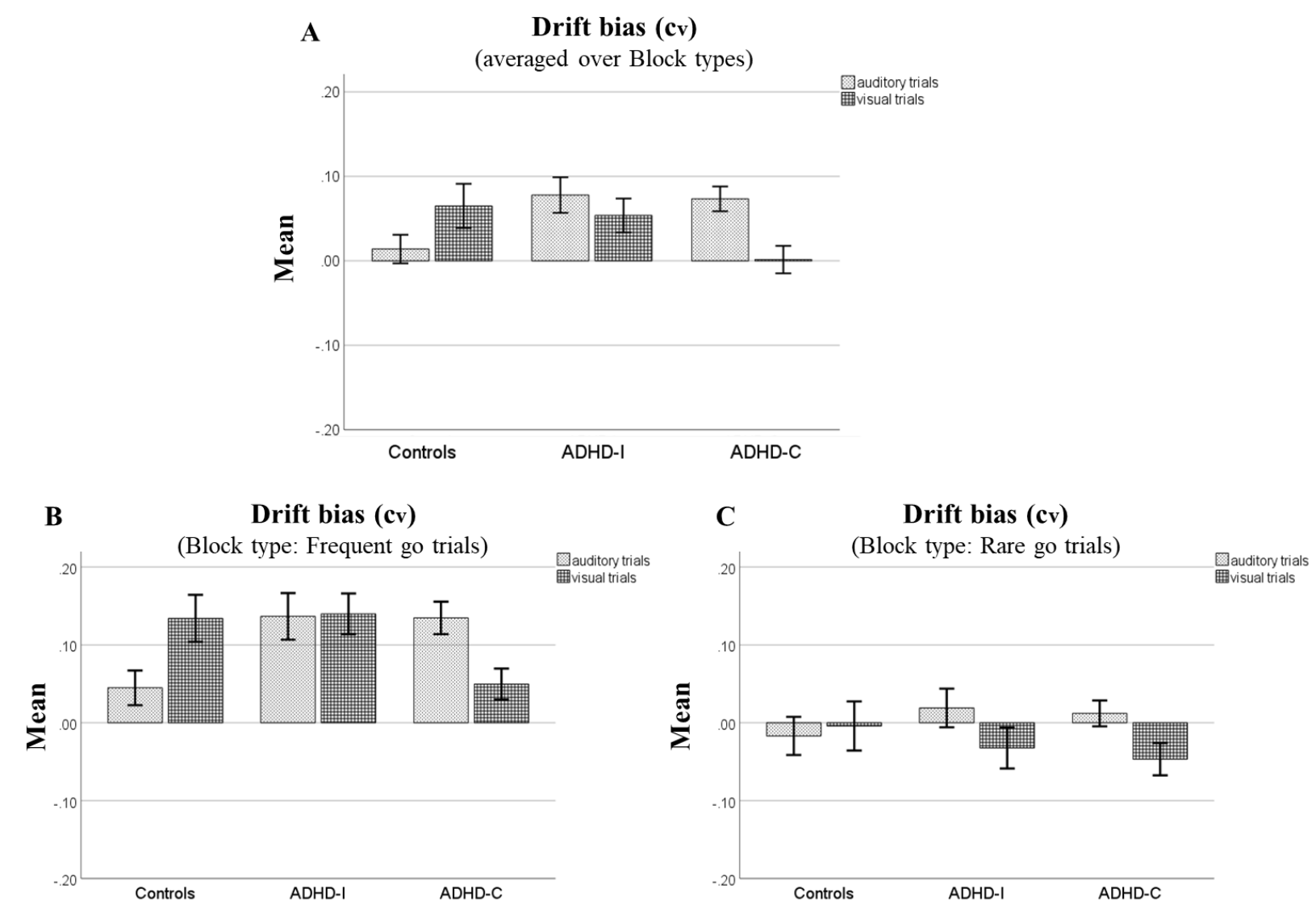

Figure 1.A. Drift biases $c_{v}$ (drift rate for go trials minus drift rate for no-go trials [absolute values; see footnote 3 in main text]) for each modality (auditory vs. visual) averaged over block type (frequent go trials vs. rare go trials). Drift bias $c_{v}$ represents context sensitivity of processes involved with information integration (with $c_{v}$ equal to zero representing the optimal level). Vertical lines represent the error bars (+/- $1 \mathrm{SE}$ ). ADHD-C was most context sensitive (largest difference in $c_{v}$ between auditory and visual trials) compared to ADHD-I and controls. For auditory trials: control had significantly smaller $c_{v}$ than ADHD-C; $t(204)=-2.51, p=0.013$ and ADHD-I; $t(204)=-2.33, p=0.021$. The difference between ADHD-C and ADHD-I was not significant; $t(204)=0.18, p=0.855$. For visual trials: control had significantly larger $c_{v}$ than $A D H D-C ; t(204)=2.27, p=0.024$. The difference between ADHD-C and ADHD-I was not significant; $t(204)=1.80, p=0.073$. The difference between control and ADHD-I was also not significant; $t(204)=0.35, p=0.729$. Figures 1.B and 1.C: Drift biases $c_{v}$ for each modality, block type, and diagnostic groups. Vertical lines represent the error bars (+/- 1 SE). Positive $c_{v}$ means that go trials had higher (faster and more accurate) drift rate than no-go trials; negative $c_{v}$ means no-go trials had higher drift rate than go trials. For visual trials from the blocks with frequent go trials: $A D H D-C$ had significantly smaller $c_{v}$ than control; $t(204)=2.56, p=0.011$ and ADHD-I; $t(204)=2.48, p=0.014$. The difference between control and ADHD-I was not significant; $t(204)=-$ $0.15, p=0.884$. For the blocks with rare go trials: there were no statistically significant group differences; all $p>0.227$.For auditory trials from the blocks with frequent go trials: control had 
significantly smaller $c_{v}$ than ADHD-C; $t(204)=-2.39, p=0.018$ and ADHD-I; $t(204)=-2.71$, $p=0.007$. The difference between ADHD-C and ADHD-I was not significant; $t(204)=0.06$, $p=0.955$. For the blocks with rare go trials: differences between diagnostic groups did not reach statistical significance; all $p>0.284$. 


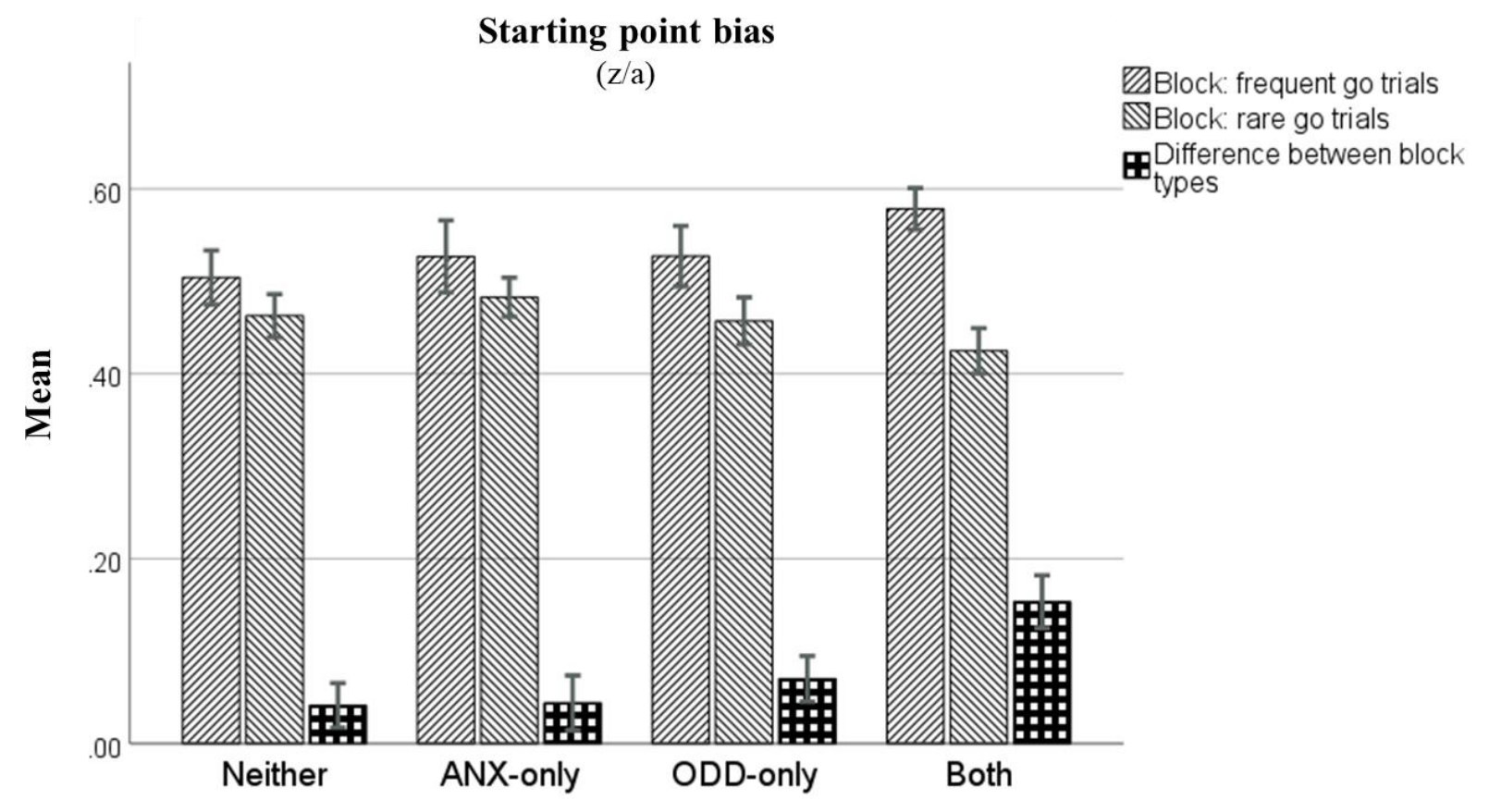

Figure 2. Starting point bias (z/a) for each block type and comorbidity group (neither, anxiety disorders only [ANX-only], oppositional defiant disorder only [ODD-only], both). Black bars =starting point bias for blocks with frequent go trials minus starting point bias for blocks with rare go trials. Vertical lines represent the error bars (+/- $1 S E)$. 


\section{Tables}

Table 1. Background characteristics for the diagnostic groups.

\begin{tabular}{|c|c|c|c|c|}
\hline & $\begin{array}{l}\text { Control } \\
(N=57) \\
\end{array}$ & $\begin{array}{l}\text { ADHD-I } \\
(N=51)\end{array}$ & $\begin{array}{l}\text { ADHD-C } \\
(N=99)\end{array}$ & Group comparisons \\
\hline $\begin{array}{l}\text { Mean }(\mathrm{SD}) \\
\text { age in years }\end{array}$ & $9(1)$ & $8(1)$ & $9(1)$ & $\begin{array}{l}\text { Control vs. ADHD-C: } n s \\
\text { Control vs. ADHD-I: ns } \\
\text { ADHD-C vs. ADHD-I: ns }\end{array}$ \\
\hline Number of females & 26 & 13 & 22 & $\begin{array}{l}\text { Control vs. ADHD-C** } \\
\text { Control vs. ADHD-I** } \\
\text { ADHD-C vs. ADHD-I: ns }\end{array}$ \\
\hline Co-morbid diagnoses ${ }^{\mathrm{a}}$ & & & & \\
\hline Neither & 0 & 21 & 23 & Control vs. ADHD-C** \\
\hline ANX & 0 & 12 & 16 & Control vs. ADHD-I** \\
\hline ODD & 0 & 7 & 30 & ADHD-C vs. ADHD-I* \\
\hline Both & 0 & 11 & 30 & \\
\hline $\begin{array}{l}\text { Mean }(\mathrm{SD}) \\
\text { T-scores on inattention }\end{array}$ & $48(6)$ & $79(8)$ & $78(8)$ & $\begin{array}{l}\text { Control vs. ADHD-C** } \\
\text { Control vs. ADHD-I** } \\
\text { ADHD-C vs. ADHD-I: ns }\end{array}$ \\
\hline $\begin{array}{l}\text { Mean (SD) } \\
\text { T-scores on hyperactivity-impulsivity }\end{array}$ & $49(7)$ & $63(11)$ & $75(12)$ & $\begin{array}{l}\text { Control vs. ADHD-C** } \\
\text { Control vs. ADHD-I** } \\
\text { ADHD-C vs. ADHD-I** }\end{array}$ \\
\hline $\begin{array}{l}\text { Child's Educational setting } \\
\text { Regular public school (N) } \\
\text { Regular public school with some } \\
\text { special classes (N) } \\
\text { Regular private/parochial school (N) } \\
\text { Home school (N) } \\
\text { Charter school (N) } \\
\text { Special school for children with } \\
\text { developmental disabilities (N) }\end{array}$ & $\begin{array}{l}2 \\
0 \\
0\end{array}$ & $\begin{array}{l}19 \\
23\end{array}$ & $\begin{array}{c}38 \\
\\
43 \\
\\
5 \\
2 \\
11\end{array}$ & $\begin{array}{l}\text { Control vs. ADHD-C** } \\
\text { Control vs. ADHD-I** } \\
\text { ADHD-C vs. ADHD-I: ns }\end{array}$ \\
\hline $\begin{array}{l}\text { Primary Caregiver's Education } \\
\text { High school/GED or less }(\mathrm{N}) \\
\text { Some college }(\mathrm{N}) \\
\text { College }(\mathrm{N}) \\
\text { Advanced degree }(\mathrm{N})\end{array}$ & $\begin{array}{c}2 \\
12 \\
21 \\
22\end{array}$ & $\begin{array}{c}2 \\
8 \\
20 \\
21\end{array}$ & $\begin{array}{l}12 \\
24 \\
39 \\
24\end{array}$ & $\begin{array}{l}\text { Control vs. ADHD-C: ns } \\
\text { Control vs. ADHD-I: ns } \\
\text { ADHD-C vs. ADHD-I: ns }\end{array}$ \\
\hline $\begin{array}{l}\text { Annual Household Income } \\
\text { Less than } \$ 23,850(\mathrm{~N}) \\
\$ 23,851-\$ 50,000(\mathrm{~N}) \\
\$ 50,001-\$ 100,000(\mathrm{~N}) \\
\text { More than } \$ 100,000(\mathrm{~N})\end{array}$ & $\begin{array}{c}2 \\
5 \\
13 \\
37 \\
\end{array}$ & $\begin{array}{c}4 \\
8 \\
22 \\
17 \\
\end{array}$ & $\begin{array}{r}9 \\
21 \\
41 \\
28 \\
\end{array}$ & $\begin{array}{l}\text { Control vs. ADHD-C** } \\
\text { Control vs. ADHD-I** } \\
\text { ADHD-C vs. ADHD-I: ns }\end{array}$ \\
\hline
\end{tabular}

Note. Group comparisons for age and T-scores are based on contrast tests. Group comparisons for number of females, co-morbid diagnoses, child's educational setting, primary caregiver's education, and annual household income are based on chi-square tests. Numbers in brackets refer

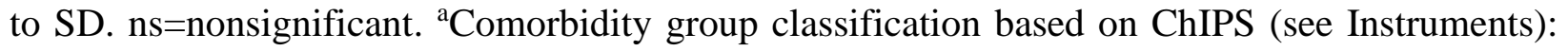
neither=neither anxiety disorders nor disruptive behavior disorders, ANX = anxiety disorders only, ODD=oppositional defiant disorders only, Both=ANX and ODD. T-scores on parent-rated inattention (AN) and hyperactivity-impulsivity (AH) (both are DSM-5 scales). ${ }^{* *} \mathrm{p}<.01$ after correction for multiple tests. 
Table 2. Means, Standard Deviations, and ANCOVA Statistics for main model parameters for diagnostic groups.

\begin{tabular}{|c|c|c|c|c|c|c|c|c|c|c|c|}
\hline \multirow[t]{2}{*}{ Variable } & \multicolumn{2}{|c|}{$\begin{array}{c}\text { Control } \\
(N=57)\end{array}$} & \multicolumn{2}{|c|}{$\begin{array}{l}\text { ADHD-I } \\
(N=51)\end{array}$} & \multicolumn{2}{|c|}{$\begin{array}{c}\text { ADHD-C } \\
(N=99)\end{array}$} & \multicolumn{5}{|c|}{ ANCOVA } \\
\hline & $M$ & $(S D)$ & $M$ & $(S D)$ & $M$ & $(S D)$ & Effect & $F$ ratio & $p$ & $d f$ & $\eta^{2}$ \\
\hline$z_{\text {bias }^{a}}$ & 0.49 & $(0.02)$ & 0.49 & $(0.02)$ & 0.50 & $(0.01)$ & G & 0.412 & 0.663 & 2 & 0.004 \\
\hline FreqGo & 0.54 & $(0.03)$ & 0.52 & $(0.03)$ & 0.55 & $(0.02)$ & GxB & 0.662 & 0.517 & 2 & 0.007 \\
\hline RareGo & 0.44 & $(0.02)$ & 0.45 & $(0.02)$ & 0.46 & $(0.02)$ & & & & & \\
\hline$T_{e r}^{a)}$ & 0.41 & $(0.02)$ & 0.43 & $(0.02)$ & 0.44 & $(0.01)$ & $\mathrm{G}$ & 0.893 & 0.411 & 2 & 0.009 \\
\hline FreqGo,vis & 0.34 & $(0.01)$ & 0.35 & $(0.01)$ & 0.35 & $(0.01)$ & $\mathrm{GxB}$ & 0.022 & 0.978 & 2 & 0.000 \\
\hline FreqGo,aud & 0.43 & $(0.02)$ & 0.46 & $(0.02)$ & 0.47 & $(0.01)$ & GxM & 0.199 & 0.820 & 2 & 0.002 \\
\hline RareGo,vis & 0.38 & $(0.03)$ & 0.41 & $(0.03)$ & 0.43 & $(0.02)$ & GxBxM & 1.842 & 0.161 & 2 & 0.018 \\
\hline RareGo,aud & 0.50 & $(0.02)$ & 0.50 & $(0.02)$ & 0.50 & $(0.02)$ & & & & & \\
\hline$v^{a)}$ & $0.26^{b)}$ & $(0.01)$ & $\left.0.19^{c}\right)$ & $(0.01)$ & 0.17 & $(0.01)$ & G & 14.057 & $<0.001$ & 2 & 0.123 \\
\hline FreqGo,vis & 0.22 & $(0.01)$ & 0.16 & $(0.01)$ & 0.13 & $(0.01)$ & GxB & 1.763 & 0.174 & 2 & 0.017 \\
\hline FreqGo,aud & 0.20 & $(0.02)$ & 0.15 & $(0.02)$ & 0.12 & $(0.01)$ & GxM & 0.417 & 0.659 & 2 & 0.004 \\
\hline RareGo,vis & 0.32 & $(0.02)$ & 0.23 & $(0.02)$ & 0.21 & $(0.01)$ & GxBxM & 0.174 & 0.840 & 2 & 0.002 \\
\hline RareGo,aud & 0.31 & $(0.02)$ & 0.23 & $(0.02)$ & 0.21 & $(0.01)$ & & & & & \\
\hline$c_{v}{ }^{a)}$ & 0.06 & $(0.02)$ & 0.06 & $(0.02)$ & $\mathbf{0 . 0 3}$ & $(0.01)$ & $\mathrm{G}$ & 1.248 & 0.289 & 2 & 0.012 \\
\hline FreqGo,vis & 0.16 & $(0.03)$ & 0.13 & $(0.03)$ & 0.04 & $(0.02)$ & GxB & 0.502 & 0.606 & 2 & 0.005 \\
\hline FreqGo,aud & 0.06 & $(0.03)$ & 0.13 & $(0.03)$ & 0.13 & $(0.02)$ & GxM & 6.662 & 0.002 & 2 & 0.062 \\
\hline RareGo,vis & -0.01 & $(0.03)$ & -0.03 & $(0.03)$ & 0.01 & $(0.02)$ & GxBxM & 6.765 & 0.001 & 2 & 0.063 \\
\hline RareGo,aud & 0.01 & $(0.03)$ & 0.01 & $(0.02)$ & -0.05 & $(0.02)$ & & & & & \\
\hline
\end{tabular}

Note. ANCOVA=analysis of variance (controlling for differences in sex, educational setting, and annual household income); presented p-values are not Bonferroni-corrected. For z bias, we applied one ANOVA with block type as within-subject variable, therefore: Bonferroni adjusted significance level equals to 0.025 (i.e., 0.05/2 conditions). For Ter, $v$, and $c_{v}$, we applied one ANOVA with block type and modality as within-subject variable, therefore: Bonferroni adjusted significance level equals to 0.004 (i.e., 0.05/[3 parameters * 4 conditions]). $\mathrm{G}=$ diagnostic groups; $\mathrm{B}=$ block type; $\mathrm{M}=$ modality. $\mathrm{z}$ bias= $\mathrm{z} / \mathrm{a}$ bias in starting point; $T_{e r}=$ nondecision time; $v=\mathrm{drift}$ rate represents the average drift rates for go and no-go trials (i.e., absolute values of drift rates for nogo trials, see footnote 3 in the main text). Supplemental Table S1 illustrates the individual drift rates for go and no-go trials; $c_{v}=$ drift rates for no-go trials (absolute values, see footnote 3 in the main text) minus drift rates for go trials; ${ }^{\text {a) }}$ values averaged over block type and modality; ${ }^{\text {b) }}$ contrast tests: control vs. ADHD-I: $t(204)=3.67 * *$; control vs. ADHD-C: $t(204)=5.87^{* *}$. ${ }^{\text {c) }}$ ADHD-I vs. ADHD-C: $t(204)=1.56$. 
Table 3. Means, Standard Deviations, and ANCOVA Statistics for main model parameters for comorbidity groups.

\begin{tabular}{|c|c|c|c|c|c|c|c|c|c|c|c|c|c|}
\hline \multirow[t]{2}{*}{ Variable } & \multicolumn{2}{|c|}{$\begin{array}{l}\text { Neither } \\
(N=44)\end{array}$} & \multicolumn{2}{|c|}{$\begin{array}{c}\text { ANX } \\
(N=28)\end{array}$} & \multicolumn{2}{|c|}{$\begin{array}{c}\text { ODD } \\
(N=37)\end{array}$} & \multicolumn{2}{|c|}{$\begin{array}{c}\text { Both } \\
(N=41)\end{array}$} & \multicolumn{5}{|c|}{ ANCOVA } \\
\hline & $M$ & $(S D)$ & $M$ & $(S D)$ & $M$ & $(S D)$ & $M$ & $(S D)$ & Effect & $F$ ratio & $p$ & $d f$ & $\eta^{2}$ \\
\hline zbias $^{a) 1)}$ & 0.48 & (0.02) & 0.50 & $(0.03)$ & 0.49 & $(\mathbf{0 . 0 2})$ & 0.50 & (0.02) & C & 0.14 & .938 & 3 & .003 \\
\hline FreqGo & 0.50 & $(0.03)$ & 0.53 & $(0.03)$ & 0.53 & $(0.03)$ & 0.58 & $(0.03)$ & CxB & 3.86 & .011 & 3 & .075 \\
\hline RareGo & 0.46 & $(0.02)$ & 0.48 & $(0.03)$ & 0.46 & $(0.02)$ & 0.42 & $(0.02)$ & & & & & \\
\hline$\left.T_{e r}^{a}\right)$ & 0.44 & $(0.02)$ & 0.42 & $(0.02)$ & 0.43 & $(0.02)$ & 0.43 & $(0.02)$ & $\mathrm{C}$ & 0.13 & .943 & 3 & .003 \\
\hline FreqGo,vis & 0.34 & $(0.01)$ & 0.36 & $(0.02)$ & 0.34 & $(0.01)$ & 0.36 & $(0.01)$ & $\mathrm{CxB}$ & 1.68 & .174 & 3 & .034 \\
\hline FreqGo,aud & 0.45 & $(0.02)$ & 0.44 & $(0.03)$ & 0.48 & $(0.02)$ & 0.47 & $(0.02)$ & CxM & 0.41 & .743 & 3 & .009 \\
\hline RareGo,vis & 0.45 & $(0.03)$ & 0.41 & $(0.04)$ & 0.41 & $(0.03)$ & 0.39 & $(0.03)$ & CxBxM & 0.20 & .896 & 3 & .004 \\
\hline RareGo,aud & 0.51 & $(0.02)$ & 0.49 & $(0.03)$ & 0.51 & $(0.02)$ & 0.49 & $(0.02)$ & & & & & \\
\hline$v^{a)}$ & 0.17 & $(0.01)$ & 0.16 & $(\mathbf{0 . 0 2})$ & 0.18 & (0.02) & 0.18 & $(0.01)$ & $\mathrm{C}$ & 0.88 & . 454 & 3 & .018 \\
\hline FreqGo,vis & 0.14 & $(0.01)$ & 0.12 & $(0.02)$ & 0.14 & $(0.02)$ & 0.15 & $(0.12)$ & $\mathrm{CxB}$ & 0.37 & .772 & 3 & .008 \\
\hline FreqGo,aud & 0.13 & $(0.02)$ & 0.12 & $(0.02)$ & 0.13 & $(0.02)$ & 0.13 & $(0.09)$ & CxM & 0.07 & .977 & 3 & .001 \\
\hline RareGo,vis & 0.21 & $(0.02)$ & 0.21 & $(0.02)$ & 0.24 & $(0.02)$ & 0.22 & $(0.18)$ & CxBxM & 1.03 & .381 & 3 & .021 \\
\hline RareGo,aud & 0.21 & $(0.02)$ & 0.20 & $(0.02)$ & 0.22 & $(0.02)$ & 0.23 & $(0.20)$ & & & & & \\
\hline$c_{v}^{a)}$ & 0.03 & $(0.02)$ & 0.04 & $(0.02)$ & 0.04 & $(\mathbf{0 . 0 2})$ & 0.04 & $(\mathbf{0 . 0 2})$ & $\mathrm{C}$ & 0.57 & .638 & 3 & .012 \\
\hline FreqGo,vis & 0.08 & $(0.03)$ & 0.08 & $(0.04)$ & 0.11 & $(0.03)$ & 0.01 & $(0.03)$ & $\mathrm{CxB}$ & 2.22 & .088 & 3 & .045 \\
\hline FreqGo,aud & 0.13 & $(0.03)$ & 0.17 & $(0.04)$ & 0.12 & $(0.03)$ & 0.12 & $(0.03)$ & CxM & 1.25 & .293 & 3 & .026 \\
\hline RareGo,vis & -0.10 & $(0.03)$ & -0.12 & $(0.04)$ & -0.04 & $(0.04)$ & -0.03 & $(0.03)$ & CxBxM & 0.21 & .890 & 3 & .004 \\
\hline RareGo,aud & -0.01 & $(0.03)$ & 0.01 & $(0.03)$ & -0.02 & $(0.03)$ & 0.06 & $(0.03)$ & & & & & \\
\hline
\end{tabular}

Note. Comorbidity group classification: Neither=neither anxiety disorders nor disruptive behavior disorders, ANX=anxiety disorders only, ODD=oppositional defiant disorders only, Both=ANX and ODD. ANCOVA=analysis of variance (controlling for differences in DSM-5-defined presentations. We did not find any significant ADHD presentations by comorbidity interactions.); $\mathrm{C}=$ comorbidity group; $\mathrm{B}=$ block type; $\mathrm{M}=$ modality. $\mathrm{z}$ bias=bias in starting point; $T_{e r}=$ nondecision time; $v=$ drift rate represents the average drift rates for go and no-go trials (i.e., absolute values of drift rates for no-go trials, see footnote 3 in the main text). Supplemental Table S1 illustrates the individual drift rates for go and no-go trials; $c_{v}=$ drift rates for no-go trials (absolute values, see footnote 3 in the main text) minus drift rates for go trials; ${ }^{\text {a) }}$ values averaged over block type and modality; Presented p-values are not Bonferroni-corrected. For $\mathrm{z}$ bias, we applied one ANOVA with block type as within-subject variable, therefore: Bonferroni adjusted significance level equals to 0.025 (i.e., 0.05/2 conditions). For Ter, $\mathrm{v}$, and $\mathrm{c}_{\mathrm{v}}$, we applied one ANOVA with block type and modality as within-subject variable, therefore: Bonferroni adjusted significance level equals to 0.004 (i.e., 0.05/[3 parameters $* 4$ conditions]). ${ }^{1)}$ Contrast tests (with Bonferroni adjustments) for changes in model parameters across block types. Group comparisons: 1 . neither vs. anxiety only (for $z: t(146)=0.07$; for $z$ bias: $t(146)=-0.07)$. 2. neither vs. ODD only (for $z: t(146)=-0.66$; for $z$ bias: $t(146)=-0.79)$. 3. neither vs. both (for $z: t(146)=-$ 
3.27; for $z$ bias: $t(146)=-3.16)$. 4. anxiety only vs. ODD only (for $z: t(146)=-0.65$; for $z$ bias: $t(146)=-0.63)$. 5 . anxiety only vs. both (for $z: t(146)=-2.96$; for $z$ bias: $t(146)=-2.72)$. 6. ODD only vs. both (for $z: t(146)=-2.48$; for $z$ bias: $t(146)=-2.25$ ). 